Voix et Images

volxetimages

\title{
Sociologie de la littérature : deux approches
}

\section{Jacques Pelletier}

Volume 11, numéro 2 (32), hiver 1986

Michel van Schendel

URI : https://id.erudit.org/iderudit/200561ar

DOI : https://doi.org/10.7202/200561ar

Aller au sommaire du numéro

Éditeur(s)

Université du Québec à Montréal

ISSN

0318-9201 (imprimé)

1705-933X (numérique)

Découvrir la revue

Citer cet article

Pelletier, J. (1986). Sociologie de la littérature : deux approches. Voix et Images, 11(2), 322-325. https://doi.org/10.7202/200561ar d'utilisation que vous pouvez consulter en ligne.

https://apropos.erudit.org/fr/usagers/politique-dutilisation/ 


\title{
Essai
}

\section{Sociologie de la littérature: deux approches}

\author{
par Jacques Pelletier, Université du Québec à Montréal
}

Les travaux en sociologie de la littérature se multiplient et se diversifient depuis quelques années au Québec. Les ouvrages publiés récemment par le

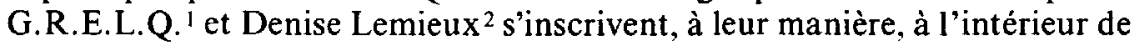
deux courants importants dans cette discipline: l'analyse institutionnelle telle que Jéveloppée par Pierre Bourdieu et ses "disciples» (cas du G.R.E.L.Q.), la sociologie des "contenus» telle que pratiquée ici par un Jean-Charles Falardeau par exemple (cas de Denise Lemieux).

L'ouvrage publié par le G.R.E.L.Q. réunit une série de monographies sur les maisons d'édition "littéraires" des années 1940 à 1960 , période durant laquelle se constitue le "champ» littéraire québécois. Dans la constitution de ce champ, les éditeurs, on le sait, jouent un rôle déterminant dans la mesure où ils orientent la production dans une certaine direction (p. 4), production qui est par ailleurs elle-même "réglée" par la commande sociale du/des public(s) lecteur(s), ce que Jacques Michon a bien montré dans son analyse du roman psychologique, roman conforme des années $1950^{3}$ et qu'il rappelle dans son texte d'introduction générale à l'étude de l'édition littéraire de cette période.

Les autres contributions réunies dans l'ouvrage se présentent sous la forme de descriptions de type monographique sur des maisons d'édition spécifiques. Il en ressort que les "grandes" maisons de la période de la guerre (Variétés, Valiquette) produisent largement pour un public «extérieur" au Québec, ce qui ne va pas sans influencer leurs choix éditoriaux se traduisant par une publication considérable d'œuvres françaises au détriment des œuvres québécoises. Cela ne présente pas toutefois que des inconvénients: d'une part le public québécois dispose d'un accès facile à la littérature française. D'autre part, grâce aux revenus générés par ces publications, ces maisons peuvent aussi éditer des auteurs québécois. C'est ainsi par exemple que Variétés publiera des ouvres d'André Giroux, de Ringuet, de Jean Simard, etc. et que Valiquette publiera des œuvres de Robert Choquette, François Hertel, Jean Narrache, etc.

Après la guerre ces maisons disparaîtront suite au regain d'activité de l'édition française. Demeurera Fides, oeuvre de propagande catholique selon l'heureuse expression d'Hélène Lafrance. Fondée en 1937, la maison existe toujours aujourd'hui, ce qui en fait vraiment un phénomène tout à fait exceptionnel dans l'histoire de l'édition québécoise. Cette longévité s'expli- 
que bien entendu dans une large mesure par la vocation même de la maison, véritable ouvre d'apostolat intellectuel soutenue par une Église toute puissante au moment de sa fondation et dans les deux décennies qui la suivent. Grâce à cela, Fides pourra prospérer et publier, outre les ouvrages de propagande catholique qui constituaient sa raison d'être, des textes littéraires québécois en grand nombre: 111 titres entre 1940 et 1960, ce qui en fait, selon Hélène Lafrance, l'éditeur québécois le plus important de cette période.

Mais ce n'est pas chez Fides, pas plus que chez Variétés ou Valiquette, qu'on trouvera des productions littéraires d'avant-garde. C'est en effet chez de petites maisons de type artisanal qu'on trouvera notamment des plaquettes de poésie d'inspiration surréaliste, aux Cahiers de la file indienne, chez Erta ou Quartz, maisons disparues aujourd'hui dont Richard Giguère et Robert Yergeau nous rappellent la courte mais significative histoire.

En somme cet ouvrage apporte une contribution intéressante et fort pertinente à la connaissance du champ littéraire québécois saisi à partir des lieux de production éditoriaux, appareils dont on ne saurait contester l'importance stratégique dans la vie littéraire d'une société.

Denise Lemieux, dans Une culture de la nostalgie, aborde la littérature d'un point de vue plus strictement "sociologique». Le titre de son ouvrage illustre d'ailleurs, on ne peut plus clairement, son propos. Ce qu'il s'agit d'étudier ici, en effet, c'est une société dans sa dimension culturelle et la littérature est d'abord considérée comme un réservoir de "documents". Choisir la littérature, écrit l'auteure, et plus particulièrement le roman, permettait d'introduire une dimension historique à cette recherche (p. 10) qui porte d'abord sur le processus de socialisation des enfants dans la société québécoise.

Dans la première partie de l'analyse - «La littérature comme idéologie» - les romans qui constituent le corpus de l'étude sont mis à contribution comme témoignages particulièrement saisissants et significatifs sur l'évolution de la famille québécoise. Leur analyse permet de dégager l'importance de la famille dans notre culture traditionnelle: lieu-refuge et point d'ancrage des valeurs auxquelles la communauté est le plus fortement attachée. La thématique récurrente de la "vieille maison", l'image archétypale du "ber" constituent autant d'illustrations d'une culture fondée sur la cohésion et l'homogénéité sociales qu'il faut sauvegarder, protéger des influences de l'extérieur - de la ville, capitaliste et cosmopolite, donc doublement menaçante: La symbolique du "restons chez nous», écrit Denise Lemieux, était la traduction, au niveau de l'imaginaire, d'une idéologie dont les principaux définisseurs participaient à des groupements voués à la défense de la langue et de la culture, celle-ci étant définie comme un monde traditionnel menacé par l'industrialisation et l'urbanisation (p. 55-56).

Dans la seconde partie de l'ouvrage - «La littérature comme phénoménologie de la socialisation" - les romans sont, cette fois, abordés comme fournisseurs d'exemples concrets du fonctionnement social et affectif de la famille québécoise. Après l'analyse des fonctions idéologiques de la famille 
donc, l'étude de son organisation interne comme univers sociétal autonome. Il s'en dégage que l'image du père, pour l'enfant, est souvent associée à l'absence, au départ (pour le travail à l'extérieur, notamment en forêt durant les mois d'hiver), alors que celle de la mère apparaît liée au monde de la maison, des valeurs d'entraide et des valeurs religieuses (p. 115). Cette observation recoupe celle effectuée naguère par Jean-Charles Falardeau quant à l'importance de la mère dans la famille québécoise, d'autant plus grande que celle-ci est souvent une associée privilégiée du curé avec qui elle forme une sorte de "couple». Si bien que cette société, d'une certaine manière, aura été longtemps plutôt matriarcale que patriarcale, du moins dans les faits, en dépit du statut objectivement (et légalement) "minoritaire» des femmes: c'est par elles que s'opérait pour l'essentiel le processus de socialisation des enfants, pour le meilleur comme pour le pire. Les textes littéraires, sur ce plan, corroborent les observations des sociologues qui ont fait enquête sur le terrain.

On voit que cet ouvrage est profondément différent de celui du G.R.E.L.Q. Son objet, c'est la société globale: la culture québécoise appréhendée à partir d'un point d'ancrage privilégié, la famille, sur laquelle les œuvres littéraires apportent des témoignages particulièrement éclairants. Le propos est donc d'abord, sinon exclusivement, sociologique. Celui qui anime le collectif G.R.E.L.Q. est sensiblement différent: d'une part l'objet d'étude est moins vaste - il s'agit d'une description d'un élément du champ littéraire québécois: le milieu éditorial de la période 1940-1960; d'autre part l'enquête sociologique ne constitue pas une fin en soi: les études empiriques sont conduites dans la perspective d'une lecture plus extensive, globalisante des textes littéraires de la période retenue. On sent bien que ces travaux ne constituent que des relais vers une interprétation plus générale de la production littéraire des années 1940-1960 et que l'analyse institutionnelle est plus un point de départ qu'un point d'arrivée.

Dans l'ouvrage de Denise Lemieux, les textes ont un statut de données et servent de points d'appui à une analyse dont le véritable objet est le social; dans l'ouvrage du G.R.E.L.Q. les lieux éditoriaux forment, avec d'autres, les conditions de production des textes qui constituent l'objet premier de l'analyse. Si bien que si, dans le premier cas, on a incontestablement affaire à un ouvrage de sociologie, dans le second, on est plutôt en présence d'une "étude littéraire" recourant à un mode d'analyse emprunté à une certaine sociologie mais pour des fins d'abord littéraires. En d'autres termes, s'il est clair que Denise Lemieux est une sociologue, il est tout aussi patent que les collaborateurs du G.R.E.L.Q. sont des «littéraires». Cette constatation n'enlève rien au mérite des ouvrages concernés; elle permet toutefois de mieux faire ressortir leur propos respectif que l'utilisation pour les qualifier du label général de «sociologie de la littérature» aurait pour effet d'oblitérer.

1. G.R.E.L.Q., l'Édition littéraire au Québec de 1940 à 1960, Sherbrooke, Département d'études françaises de l'Université de Sherbrooke, 1985, 217 p. (Coll. "Cahier d'études littéraires et culturelles"). 
2. Denise Lemieux, Une culture de la nostalgie, Montréal, Boréal Express, 1984, 245 p.

3. Jacques Michon, «Esthétique et réception du roman conforme», in Structure, idéologie et réception du roman québécois de 1940 à 1960, Sherbrooké, Département d'études françaises de l'Université de Sherbrooke, 1979, p. 4-20 (Coll. "Cahiers d'études littéraires et culturellesn). 